\title{
Peripheral field stimulation suppresses flicker but not pattern detection in foveal targets
}

\author{
R. T. MARROCCO, M. A. CARPENTER, S. E. WRIGHT, and R. A. DORAN \\ Institute of Neuroscience, University of Oregon, Eugene, Oregon
}

This report examines the effects of peripheral visual stimulation on foveal target thresholds. The foveal targets were luminance-modulated sine-wave gratings (subtending $2^{\circ}$ arc), presented with or without a phase-alternation of $1 \mathrm{~Hz}$. The peripheral stimulus was a $20^{\circ}$ black-white radial or linear, square-wave grating. The radial grating contained a $2^{\circ}$ blank central area and appeared either at rest or in radial motion at $5^{\circ} / \mathrm{sec}$. The linear grating contained either a $2^{\circ}$ or an $8^{\circ}$ central blank aperture and appeared either at rest or in oscillation by one-half cycle at $4 \mathrm{hz}$. Subjects always viewed the foveal target with their left eyes. The peripheral stimulus was presented to the left eye for monocular viewing but to the right eye for dichoptic viewing. For dichoptic viewing, the radial but not the linear grating increased the thresholds for counterphase but not stationary target gratings. For monocular viewing, both peripheral stimuli had threshold elevating effects on counterphase but not stationary targets. Results are discussed in terms of the binocular control of foveal monocular sensitivity.

Visual stimulation of the peripheral retina with a rapidly displaced linear grating reduces foveal sensitivity to nearthreshold spot stimuli (Breitmeyer \& Valberg, 1979; Marrocco, Carpenter, \& Wright, 1985; Valberg \& Breitmeyer, 1980). Similarly, recent experiments have suggested that radial peripheral gratings reduce the visibility of foveally viewed counterphase gratings (Marrocco et al., 1985). A moderate rate of peripheral temporal stimulation (i.e., radial-grating velocity) appears to be important to obtain the visibility reduction (Breitmeyer, Valberg, Kurtenbach, \& Neumeyer, 1980; Marrocco et al., 1985).

The functional importance of these spatial interactions is unclear. According to one hypothesis, foveal-sustained, or pattern-detecting, channels are inhibited by transient, flicker-detecting peripheral channels during eye movements to facilitate analysis of patterns during subsequent fixation (Breitmeyer \& Valberg, 1979). However, primate physiological work (de Monasterio \& Schein, 1980) has shown that transient $(\mathrm{Y})$ neurons as well as sustained (X) neurons have foveal receptive fields. Does peripheral stimulation also affect transient foveal channels?

To answer this, we must distinguish behaviorally between the actions of each channel with appropriately chosen stimuli. We adopted a slight modification of the stimuli used by Tolhurst and his colleagues (Kulikowski \& Tolhurst, 1973; Tolhurst, 1973), employing stationary sine-wave gratings to preferentially excite the pattern channel and counterphase sine-wave gratings to stimulate the flicker channel. We examined the effect of peripheral

We thank N. Graham and F. Attneave for helpful criticisms of the manuscript. This work was supported in part by NSF Grant BNS 8207531 and NIH Grant EY 05693. The authors' address is Institute of Neuroscience, University of Oregon, Eugene, OR 97403. stimulation on foveal detection of counterphase and stationary gratings. Because previous work had suggested that different rules might affect monocular and dichoptic spatial interactions (e.g., Johnson \& Enoch, 1976; Marrocco et al., 1985), we tested both types of viewing condition.

According to the eye-movement hypothesis (Breitmeyer \& Valberg, 1979), the rapid stimulus displacement of the peripheral linear grating mimics the effects of saccadic eye movements. We therefore also asked whether the inhibition of foveal sensitivity was limited to rapid movement. To test this, we compared the rapid displacement of peripheral linear gratings with the slow rotation of a radial grating. A preliminary account of these results has appeared elsewhere (Marrocco \& Carpenter, 1983).

\section{METHOD}

\section{Subjects}

Three experimentally naive subjects and 1 experienced subject (R.T.M.) were used for these experiments. Each subject was carefully tested by an optometrist (S.E.W.) for refractive errors, resting vergence angle, and static stereoacuity. Each subject had 20/20 vision or wore corrective lenses for the experiments. Artificial pupils were not used. Additional data have been collected from 5 additional naive subjects. Their results, which replicate the present data in virtually all respects, are not included in this report.

\section{Apparatus and Stimuli}

Vertical sine-wave-grating stimuli were produced electronically on the screen of a Tektronix 604 monitor (P4 phosphor). The mean luminance of the screen was $9 \mathrm{~cd} / \mathrm{m}^{2}$, as measured by an EG\&G photometer. We measured the peaks and troughs of the sine waves and computed contrast as the quotient of the differences divided by the sums of the maximum and minimum luminances. The linearity of contrast changes that occurred with changes in input voltage was measured by masking the photometer detector lens with a slit of approximately the width of a single light or dark bar. This proce- 
dure underestimates the true contrast by about $30 \%$ but does not differentially affect treatment conditions. Voltage changes to the CRT produced linear changes in contrast within $0.1 \%$ over a range of contrast between 0.005 and 0.125 .

A circular aperture in a white cardboard surround limited the extent of the display to $2^{\circ}$. The dimensions of the field surrounding the aperture were $10^{\circ} \times 18^{\circ}$; this field was uniformly illuminated with an independent light source at the mean luminance of the gratings. The light was prevented from illuminating the CRT screen by suitable masking.

Eight spatial frequencies (0.9-10.0 cycles/deg (cpd) were used in these tests. The lowest obtainable frequency was dictated by the use of a $2^{\circ}$ aperture ( 2.2 cycles visible); the highest was limited by the resolution of the monitor and our linear contrast generating circuit. Although these choices leave the lowest and highest frequencies untested, they span the region of maximum sensitivity in human vision. For counterphase stimulus experiments, stimuli were phase-alternated by a $1.0-\mathrm{Hz}$ square wave. For stationary test stimuli, the counterphase function was removed.

The radial-grating stimulus was produced photographically from black-ink drawings on acetate sheets and was rear-projected onto a 3M Polacoat tangent screen. The space-averaged luminance of the grating was $27.2 \mathrm{~cd} / \mathrm{m}^{2}$. The bright bars were $54.4 \mathrm{~cd} / \mathrm{m}^{2}$ and the dark bars were $0.78 \mathrm{~cd} / \mathrm{m}^{2}$ for a contrast of $97 \%$. The color temperature of the bright bars $\left(6,000^{\circ} \mathrm{K}\right)$ was different from that of the bars produced on the CRT screen $\left(6,300^{\circ} \mathrm{K}\right.$, Tektronix special phosphor). The radial grating had an outer diameter of either $8^{\circ}$ or $20^{\circ}$ and a black central aperture of $2.0^{\circ}$. The grating had 24 sectors, each subtending a visual angle of $12^{\prime}$ arc at the 'hub" and $7.0^{\circ}$ arc at the "rim." The transparency was mounted on a gimbal that could be driven by an electric motor and belt pulley. When rotating, the vanes at the hub moved at $7.5^{\circ} \mathrm{arc} / \mathrm{sec}$. The temporal frequency at which the vanes passed any given point on the screen was $5 \mathrm{~Hz}$. Both displays were $57 \mathrm{~cm}$ from the observer's eyes.

For monocular experiments, the subjects, with right eye patched, used the left eye to view the sine waves in the center of the radial grating through a $75 \%$-reflectance $/ 25 \%$-transmittance beam splitter. After transmission losses, the mean luminance of each display was $6.7 \mathrm{~cd} / \mathrm{m}^{2}$. Fixation was steadied by the presence of a dark square subtending $10^{\prime}$ arc in the geometric center of the display.

For dichoptic experiments, the subjects used the left eye to view the 604 display reflected from a front-surface mirror. The mirror was circular and subtended $2^{\circ}$ arc. The subjects used the right eye to view the radial grating. The luminance of the radial gratings was reduced with neutral-density filters during dichoptic viewing to maintain equal peripheral luminances with the monocular condition. The subject fused the targets such that the sine-wave display appeared at the center of the radial grating. To reduce fusional instability, we employed fixation aids. One dark ring surrounded the sine-wave display and a second outlined the central $2.0^{\circ}$ radial-grating aperture. In addition, we adjusted the location and angle of the frontsurface mirror to approximate the resting vergence angle of each subject, thus minimizing corrective fusional movements. A trial lens was placed in front of each eye, when necessary, to produce the best image of each stimulus. $A$ chin- and foreheadrest and head straps were used to stabilize the head during threshold adjustments. A schematic drawing of the apparatus has been published previously (Marrocco et al., 1985).

\section{Procedure}

At the start of each experimental session, the subjects were adapted for $10 \mathrm{~min}$ to an unstructured field whose luminance was equal to the luminance of the radial grating $\left(6.7 \mathrm{~cd} / \mathrm{m}^{2}\right)$. They were then presented with randomized series of trials in which the counterphase test grating of fixed spatial frequency was paired with the stationary or moving radial-grating surround. One "run" thus con- sisted of 16 threshold settings. Two or three complete repetitions were completed per experimental session, and the settings were averaged across runs. Sessions for counterphase and stationary test gratings were scheduled on alternate days. A second series of experimental sessions using the same protocol was devoted to data collection with the linear surround. The total number of observations was held constant for each subject.

The subjects used the method of adjustment to bring the linear grating to threshold. The subjects were instructed to adopt a flicker criterion for detection; that is, they were told to be aware of any flicker or movement in the pattern. One series of threshold adjustments with the stationary and moving gratings was determined by increasing the contrast from zero; a second series was determined by decreasing the contrast from a suprathreshold value (usually about $10 \%$ ) to threshold. The average of the ascending and descending series was recorded for later analysis. The data presented in this report represent means and standard errors from five experimental sessions. Each data point thus represents $\mathbf{4 0}$ threshold settings.

There was a substantial learning effect in this experiment. Typically, the variance of the settings was large at the beginning of the study. The data presented here were obtained from the subjects after their responses had stabilized.

\section{RESULTS}

We first sought to learn how the contrast sensitivity for $2^{\circ}$ counterphase targets varied with spatial frequency. For these experiments the peripheral stimulus was an unstructured field with a mean luminance of $6.7 \mathrm{~cd} / \mathrm{m}^{2}$ at the subject's cornea. Averaged results for all subjects with monocular viewing (right eye occluded) are shown in Figure 1. The curve labeled $S$ represents thresholds for stationary sine-wave targets, and the curve labeled $\mathrm{C}$ shows the counterphase-stimulus thresholds. Several points are notable. First, the $C$ curve is low-pass in form, whereas the $S$ curve is band-pass. Second, the $C$ curve shows a higher absolute contrast sensitivity for low spatial frequencies than does the $S$ curve. Third, the sensitivity of the $S$ curve is higher than that of the $C$ curve for spatial frequencies above $3 \mathrm{cpd}$. These results closely parallel those found by previous investigators (e.g.,

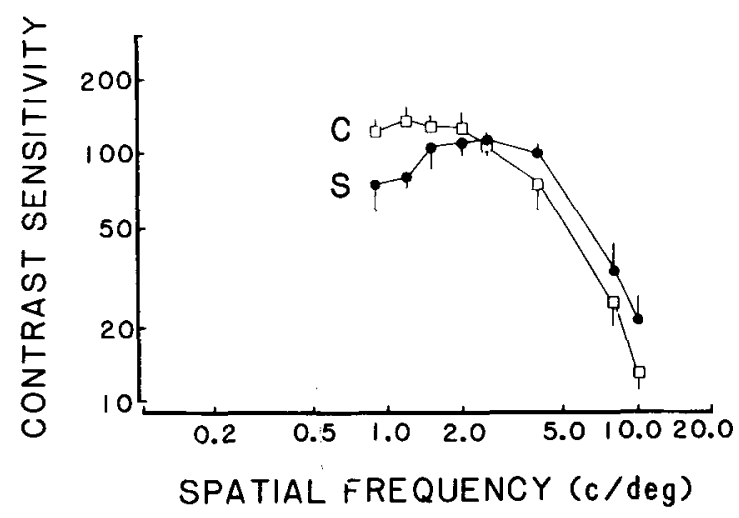

Figure 1. Comparison of contrast sensitivities for counterphase (C) and stationary (S) sine-wave gratings viewed foveally with the left eye. The right eye viewed a homogeneous field of mean huminance equivalent to the mean luminance of the targets in the left eye. Error bars represent 1 standard error of the mean. See text for further details. 


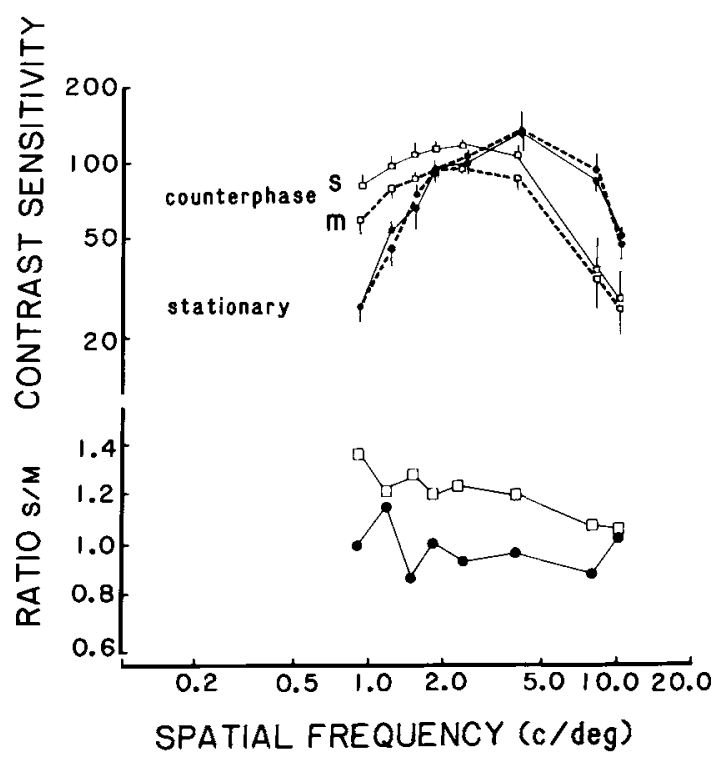

Figure 2. Comparison of contrast sensitivities for counterphase and stationary sine-wave targets seen with the left eye. The right eye viewed either the stationary (s) or spinning (m) radial grating in its peripheral field. Dichoptic viewing was used. The rations of the thresholds for the $s$ and $m$ conditions for each type of test target are shown in the lower half of the figure. Error bars represent 1 standard error of the mean.

Kulikowski \& Tolhurst, 1973), and we take these curves as the signatures of the flicker and pattern systems.

We then assessed the effect of the radial grating seen with the right eye on the thresholds for counterphase and stationary targets seen with the left eye (dichoptic viewing). For either target, the radial grating was at rest (s) or spinning at $5^{\circ} \mathrm{arc} / \mathrm{sec}(\mathrm{m})$. The averaged results of these experiments for all subjects are shown in Figure 2. Open squares show the contrast sensitivities for counterphase grating targets, solid circles show sensitivities for stationary targets. The ratios of the stationary and moving peripheral-stimulus threshold sensitivities are shown in the lower panel of Figure 2. Ratios above unity suggest that the moving stimulus elevated thresholds; ratios below unity indicate that the moving peripheral stimulus lowered thresholds. Two findings are notable. First, the characteristic shapes of the curves for the flicker and pattern systems are evident, with the crossover occurring at about 3 cpd. Second, movement of the radial grating has a larger effect on the sensitivities for counterphase than for stationary test gratings. A repeated measures analysis of variance showed a significant overall elevation of counterphase-target but not of stationary-target thresholds $[F(1,3)=10.6, p=.047]$, but an insignificant conditions $\times$ spatial frequency interaction $(p>.12)$. Singlepolynomial contrasts calculated for counterphase gratings showed that all ratios except those for 10.0-cpd stimuli were significantly elevated above unity. Therefore, the primary effect seems to be an elevation of thresholds for flicker detection.
To determine whether the pattern of peripheral stimulation was important in determining the thresholds for foveal stimuli, we replaced the radial grating with a 0.2 cpd linear grating (contrast $=94 \%$ ), whose bars were vertically oriented. The grating was excluded from a central $2^{\circ}$ aperture. Test targets of $2^{\circ}$, when fused binocularly, were viewed with a stationary or oscillating linear peripheral stimulus. The optical oscillation, a half-cycle grating displacement, was produced by supplying the galvanometer with a 4-Hz triangle wave. Figure 3 illustrates the averaged results for all subjects during dichoptic viewing. The characteristic shapes of the pattern- and flickersystem threshold curves are evident. For all subjects, however, no systematic differences between stationary and oscillating peripheral gratings are generally evident for either test grating. The ratios shown in the lower panel are generally closer to unity than those seen using the radial peripheral stimulus. For stationary targets, the mean at 8.0 cpd was significantly below unity $[t(3)=3.5, p<$ $.05]$. None of the counterphase-target thresholds was significantly different from unity $[F(1,3)=1.09, p=.37]$. These results suggest that the oscillation of the peripheral linear grating has little, if any, effect on flicker or pattern thresholds. No significant differences were found between the $2^{\circ}$-arc and the $8^{\circ}$-arc central-aperture condition (not shown).

To compare our results with previous work (Breitmeyer \& Valberg, 1979; Derrington, 1984), we also tested whether either peripheral stimulus has thresholdmodulating effects when viewed monocularly. Threshold ratios for these experiments and the foregoing dichoptic experiments are shown in Figure 4. The oscillating linear grating produced relatively large threshold increases for

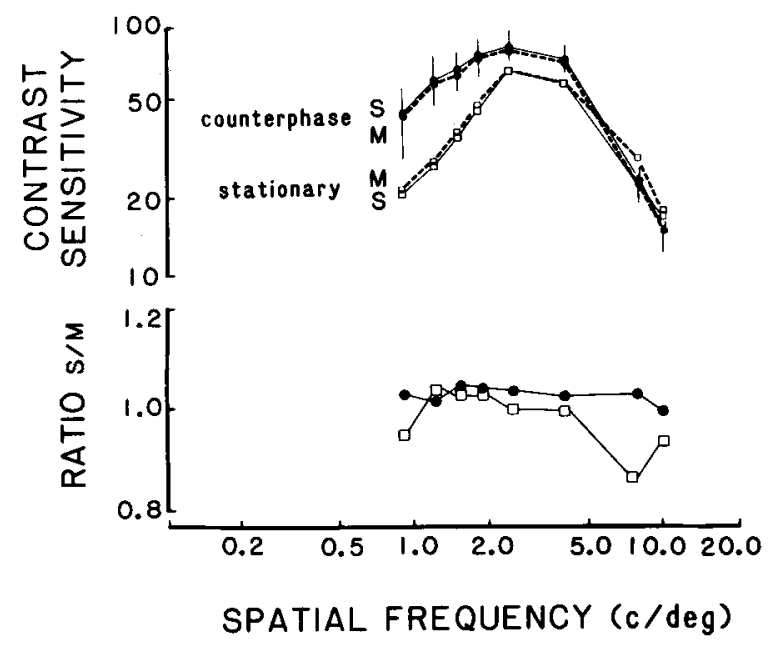

Figure 3. Comparison of contrast sensitivities for counterphase and stationary sine-wave targets seen with the left eye. The right eye viewed either a stationary (S) or oscillating (M) linear grating in its peripheral field. Error bars represent 1 standard error of the mean. Dichoptic viewing was used. The ratios of the threshold for the $S$ and $M$ conditions for each type of test target are shown in the lower half of the figure. See text for further details. 


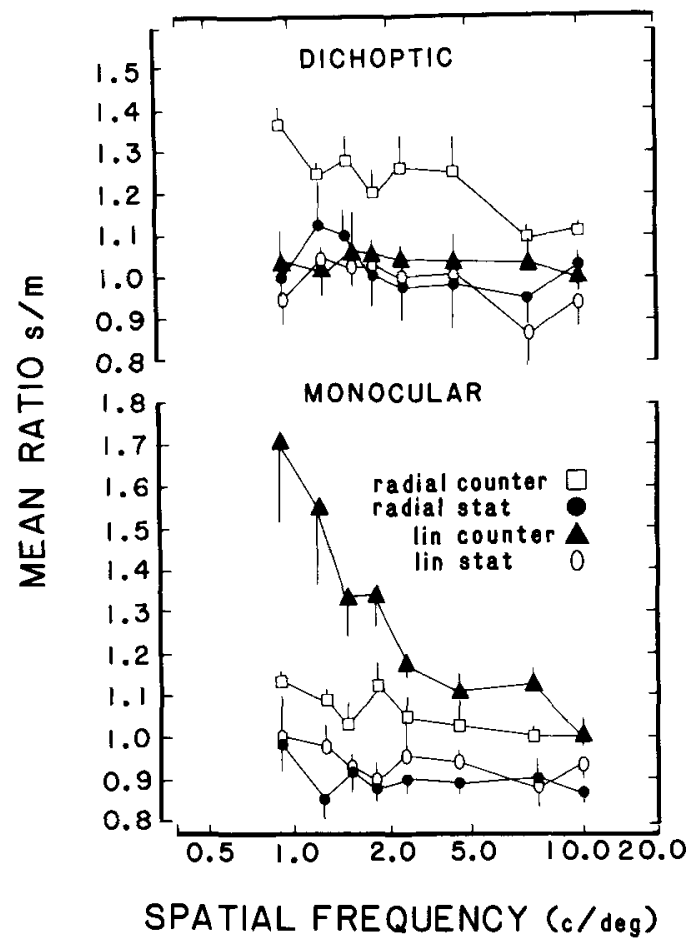

Figure 4. Comparison of ratios of $s$ to $m$ conditions for four dichoptic (top) and four monocular (bottom) viewing conditions: open squares, radial-grating peripheral stimulus, counterphase test stimulus; open circles, linear-grating peripheral stimulus, stationary test stimulus; filled circles, radial-grating peripheral stimulus, stationary test stimulus; triangles, linear-grating peripheral stimulus, counterphase test stimulus. Each point is the mean ( \pm 1 standard error) threshold for all subjects and represents 20 measurements per subject.

the low-spatial-frequency counterphase-grating targets, in agreement with previous results (Derrington, 1984; Marrocco et al., 1985) but little or no effect on the stationary test grating (lower Figure 4, open circles; see also Marrocco et al., 1985). For counterphase targets, there was a significant overall threshold elevation $[F(3,9)=16.6$, $p<.05]$. No stationary target had a significantly altered threshold $(p>.06)$.

The radial grating produced significant overall threshold elevations for counterphase stimuli $(p<.05$; lower Figure 4, open squares) but a significant overall threshold decrease $(p<.04)$ for stationary sine-wave gratings (filled circles). With the exception of the counterphase linear-grating condition, there is only a slight tendency for the monocular effects to be spatial-frequency selective, with smaller effects seen predominantly at high spatial frequencies.

\section{DISCUSSION}

The main findings of the present study show that moving peripheral stimuli produce small, but statistically significant, increases in contrast-sensitivity thresholds for foveally viewed, low-spatial-frequency counterphase stimuli. Stationary stimuli are affected to a much smaller extent. The peripheral stimulus that causes the most significant threshold elevation is the linear grating for monocular viewing and the radial grating for dichoptic viewing. Thus, the pattern of peripheral stimulation is important. The following discussion evaluates potential sources of error in our data and considers our results in light of previous findings.

Did the stationary and counterphase targets selectively stimulate the pattern and flicker channels, respectively? In the strict sense, the answer is probably no. The main difference between the channels in our experiments was a differential sensitivity to low and high spatial frequencies. However, the reduction of our test-field size to $2^{\circ}$ produced aperture and edge effects that added many other spatial frequencies to the nominal frequencies produced by the electronic generator. Thus, the regions in which the thresholds of the two systems are similar probably represent a combined effect of both. However, the differences between threshold curves in the best case (e.g., see Figure 2) approach an order of magnitude and are likely to represent predominantly the action of one system.

Did the use of criterion measures and the method of adjustment produce an accurate assessment of the sensitivities of the flicker and pattern channels? Burbeck (1981) recently answered this question in the negative. Using criterion-free methods, she found that the sensitivity of the flicker system was always less than that for the pattern system and that the differences were greatest at high spatial frequencies. The differences between her data and ours are quite minor and predictable. Her Figure 1 shows that for low spatial frequencies, viewed on a screen subtending an $8^{\circ}$ arc, the pattern sensitivity slightly exceeds the flicker sensitivity. However, our test target subtended only $a 2^{\circ}$ arc. The effect of reducing the size of the test target was to lower the sensitivity for detecting flicker in all spatial frequencies below 2 cpd by two- to threefold (Marrocco et al., 1985). Pattern thresholds in this range were affected by a factor of about 1.2-1.5. Thus, the differences for low spatial frequencies can be accounted for on the basis of target area.

A comparison of the two sets of data with the targetarea difference factored in suggests that pattern sensitivities at high spatial frequencies are almost two octaves higher than flicker sensitivities (Burbeck, 1981). Our results suggest comparable sensitivity differences (e.g., Figure 3) under some conditions, in contrast to much smaller differences reported previously (Kulikowski \& Tolhurst, 1973). Thus, we conclude that the differences between the criterion and criterion-free measures are not major when small test targets are used.

Can our results be attributed to possible fusional instabilities? Subjects unable to maintain fusion of the dichoptic stimuli might be expected to show elevated thresholds for the test stimuli. This would result primarily from the radial and test stimuli's falling on corresponding points of the retinas, creating binocular stimulation. The thresholds of binocular neurons to the test stimuli 
would probably be elevated by exposure to the peripheral stimulus. However, it is unlikely that fusional difficulties could account for the elevated thresholds of counterphase stimuli, because (1) we took special precautions to facilitate fusion (see Method), (2) subjects reported no difficulty maintaining steady fusion, and (3) the unsteadiness would be expected to influence the results of the linear-grating experiments as well as the results for stationary test stimuli. As shown in Figures 2-4, no significant elevations were found for the latter conditions. The failure of the radial grating to affect thresholds of stationary stimuli also would rule out possible artifacts produced by cyclorotatory movements caused by the rotating peripheral stimulus and potential threshold elevations caused by binocular rivalry.

Previous investigations have used radial gratings to stimulate the visual field under dichoptic viewing (Enoch, Lazarus, \& Johnson, 1976; Johnson \& Enoch, 1976). Although no spatial interactions with foveal targets were observed, test targets were flashing spots and both stimuli were restricted to the fovea, making it difficult to compare the results of the two studies.

Breitmeyer and his colleagues (Breitmeyer \& Valberg, 1979; Valberg \& Breitmeyer, 1980) reported that linear gratings raised the threshold for flashing spots viewed with the same eye. If we assume that the flashing stimulus stimulates the flicker system more than the pattern, then our results are comparable to theirs. However, although spatial interactions between linear gratings and counterphase targets are inhibitory, they are not globally inhibitory in the spatial-frequency domain. That is, they affect low and medium spatial frequencies more than they affect the high ones.

Although there is general agreement that the detection of counterphase grating targets at low spatial frequencies is mediated by transient, or pattern-detecting, channels (Kulikowski \& Tolhurst, 1973; Tolhurst, 1973), there is considerable disagreement as to the underlying neural mechanisms. Several psychophysical and physiological reports have concluded that the low-spatial-frequency flicker channel is supported by a mixture of signals from $\mathrm{X}$-like and Y-like mechanisms (Essock \& Lehmkuhle, 1982; Frascella \& Lehmkuhle, 1984; Lennie, 1980; Wood \& Kulikowski, 1978). For the current findings, the composition of the channels mediating pattern and flicker is not as important as the consistency with which they are affected.

It is somewhat puzzling that the most effective peripheral stimulus for monocular viewing should be ineffective during dichoptic viewing. Reasonable hypotheses for the radial grating's greater suppression can be found in the relative strength of binocular versus monocular inhibition in the visual cortex (e.g., Hess, Negishi, \&
Creutzfeldt, 1975). Alternatively, differences between retinal and cortical periphery effects may explain the discrepancies. We are currently designing experiments to help decide between these hypotheses.

\section{REFERENCES}

Breitmeyer, B. G., \& Valberg, A. (1979). Local, foveal inhibitory effects of global, peripheral excitation. Science, 203, 463-465.

Breitmeyer, B. G., VAlberG, A., Kurtenback, W., \& NeUmeyer, C. (1980). The lateral effect of oscillation of peripheral luminance gratings on the foveal increment threshold. Vision Research, 20, 799-805.

BuRBECK, C. A. (1981). Criterion-free pattern and flicker thresholds. Journal of the Optical Society of America, 71, 1343-1350.

de Monasterio, F. M., Schein, S. J. (1980). Ptotan-like spectral sensitivity of foveal $Y$ ganglion cells of the retina of the macaque monkey. Joumal of Physiology, London. 299, 385-396.

DeRRINGTON, A. M. (1984). Spatial frequency selectivity of remote pattern masking. Vision Research, 24, 1965-1968.

Enoch, J. M., Lazarus, J. Johnson, C. A. (1976). Human psychophysical analysis of receptive field-like properties: I. A new transientlike visual response using a moving windmill (Werblin-type) target. Sensory Processes, 1, 14-32.

Essock, E. A., LenMkUhLE, S. (1982). The oblique effects of pattern and flicker sensitivity: Implications for mixed physiological input. Perception, 11, 441-455.

Frascella, J., LehmkuHle, S. (1984). An electrophysiological assessment of $\mathrm{X}$ - and $\mathrm{Y}$-cells as pattern and flicker detectors in the dorsal lateral geniculate nucleus of the cat. Experimental Brain Research, 55, 117-126.

Hess, R., Negishy, K., Creutzfeldt, O. D. (1975). The horizontal spread of intracortical inhibition in the visual cortex. Experimental Brain Research, 22, 415-419.

JoHNSON, C. A. , ENOCH, J. M. (1976). Human psychophysical analysis of receptive field-like properties: III. Dichoptic properties of a new transient-like psychophysical function. Vision Research, 16, 1463-1470.

KuLkowski, J. J., \& TolHuRst, D. J. (1973). Psychophysical evidence for sustained and transient neurons in the human visual system. Joumal of Physiology, London, 232, 149-162.

LeNnIE, P. (1980). Perceptual signs of parallel pathways. Philosophical Transactions of the Royal Society of London, Series B, 290, 23-37.

Marrocco, R. T. CArpenter, M. A. (1983). Spatial contrast sensitivity: Effects of peripheral visual stimulation during monocular and dichoptic viewing. Society for Neuroscience Abstracts, 7, 211.

Marrocco, R. T., Carpenter, M. A., \& Wright, S. E. (1985). Spatial contrast sensitivity: Effects of peripheral field stimulation during monocular and dichoptic viewing. Vision Research, 25, 917-924.

TolHURST, D. J. (1973). Separate channels for the analysis of the shape and the movement of a moving visual stimulus. Joumal of Physiology, London, 231, 385-402.

VALBERG, A., BREITMEYER, B. G. (1980). The lateral effect of oscillation of peripheral luminance gratings: Test of various hypotheses. Vision Research, 20, 789-798.

Wood, I. C. J., Kucrkowski, J. J. (1978). Pattern and movement detection in patients with reduced visual acuity. Vision Research, 18, 331-334.

(Manuscript received April 15, 1985; revision accepted for publication March 26, 1987.) 